\title{
Artificial Surface Tension to Stabilize Captured Shockwaves
}

\author{
Philip. L. Roe *and Keiichi Kitamura ${ }^{\dagger}$
}

\begin{abstract}
This paper proposes (yet another) method for ameliorating the tendency of many Euler and Navier-Stokes codes to produces anomalous captured shockwaves at very high Mach numbers. The proposal is at the PDE level, and therefore in principle applicable to any type of grid. It is based on an analogy with surface tension.
\end{abstract}

\section{Introduction}

Computationally captured shockwaves, especially at very high Mach number, often display anomalous behavior not encountered in nature. Figure 1 shows three of the most notorious examples. The passage of a normal shock over a slightly perturbed mesh was observed to be unstable by Quirk. ${ }^{11}$ However, it was subsequently discovered that neither the mesh perturbation nor the shock motion was always needed. ${ }^{4}$ Diffraction of a plane shock wave by a wedge often causes a kink in the Mach stem, which does not properly intersect the wedge surface. ${ }^{12}$ Perhaps most notorious is the so-called carbuncle phenomenon, first reported by Peery and Imlay. ${ }^{10}$

These anomalies are perplexing, not least because they are strongly related to genuine physical solutions of the Euler equations. When they appear in steady solutions, the residuals are frequently very small, ${ }^{6,9}$ indicating that a weak solution has been achieved, and moreover the shockwaves are compressive and entropysatisfying. Versions of the carbuncle have been demonstrated experimentally at high Reynolds number, ${ }^{7}$ and even proposed as practical devices to reduce drag and heat transfer. ${ }^{8}$

There are even anomalies in one dimension. ${ }^{2,3}$ Astonishingly, Godunov's method has no solution for a steady one-dimensional shock at Mach numbers greater than 6.0 (if $\gamma=7 / 5$ ) unless the shock resides within a certain range of locations relative to the mesh.

It has been hypothesized that the failure is in part thermodynamic, associated with the fact that even the "exact" solution of the Riemann problem does not strictly enforce the Second Law locally. However, Kitamura et $\mathrm{al}^{6}$ demonstrated that while flux functions free of this defect may be satisfactory in one dimension, the carbuncle can return in higher dimensions. It seems that some intrinsically multidimensional approach needs to be taken, in addition to correcting the thermodynamics.

\section{A New Proposal}

One seemingly universal observation about the anomalous shocks is that their shapes are less smooth than those in the "true" solutions. They may develop spurious kinks, or they may tend to track irregularities in the computational grid. This prompted the search for a form of dissipation that would have the effect of smoothing captured shocks while leaving other features of the flow unaffected. The smoothing is of course along the shock The object is to bestow additional stability on captured shocks, so that they behave more like real ones. For example, an initially corrugated ideal shock returns to a planar state like $t^{-1},{ }^{13}$ but an initially corrugated captured shock may diverge. ${ }^{6}$ This is apparently because on any grid, however fine, a captured shock has internal structure in the form of intermediate states. These are artificial, but serve to locate the shock in space. They are a necessary consequence of the shock-capturing philosophy, but may have a destabilizing effect.

If this were so, it would be reasonable to counteract it by introducing another artificial effect that enhances stability. The proposal developed here is inspired by the idea of surface tension. There is a pressure difference,

*Professor,Department of Aerospace Engineering, University of Michigan, Ann Arbor, MI 48109, USA, Fellow AIAA

${ }^{\dagger}$ Doctoral student, Department of Aerospace Engineering, Nagoya University, Japan, Member AIAA 

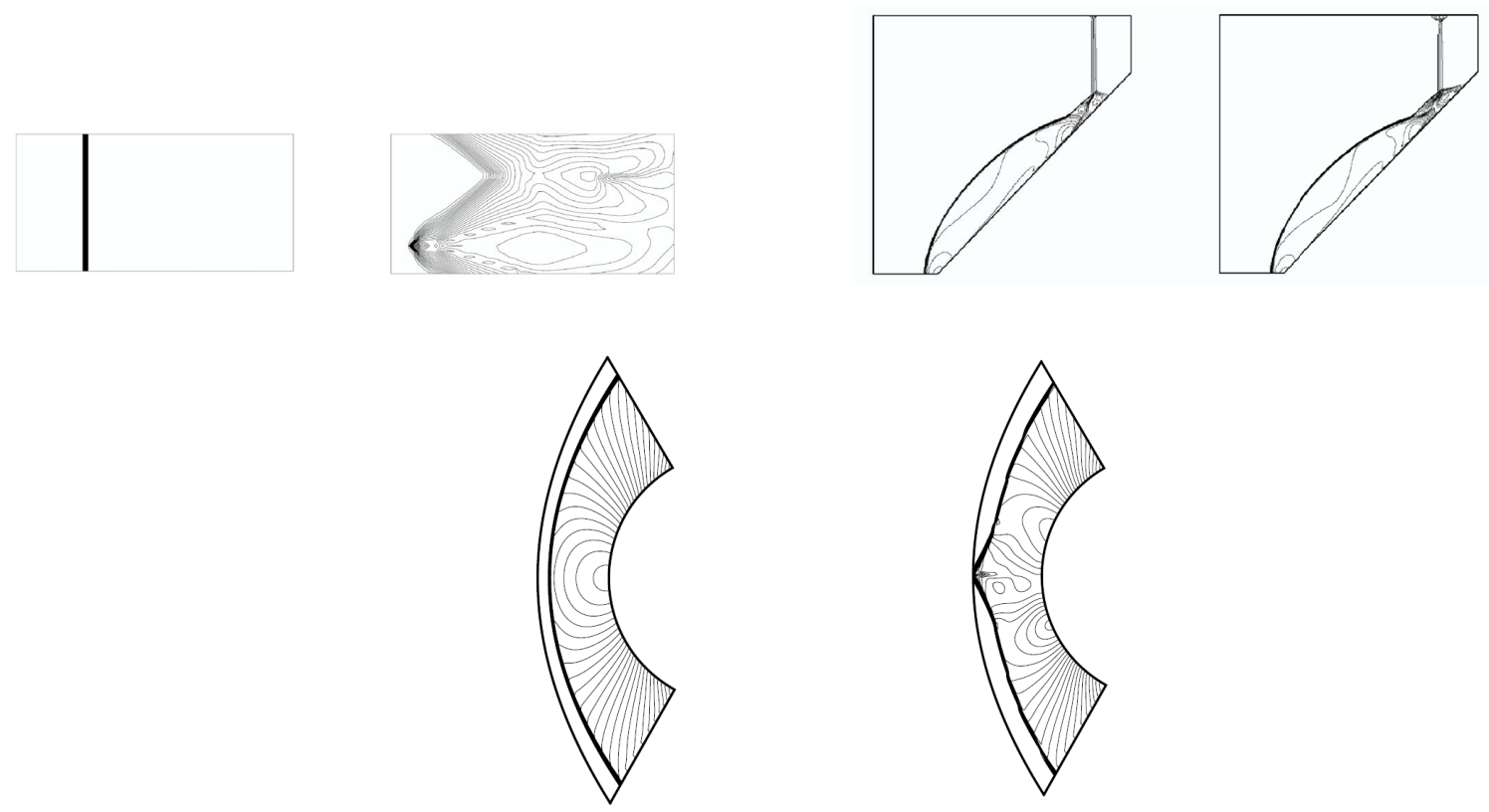

Figure 1. Regular(left) and anomalous(right) versions of three notorious problems posed for the Euler equations. Top left, a stationary shock wave in a channel; top right, diffraction of a shock by a wedge, bottom, flow past a circular cylinder.

across any interface between dissimilar fluids, given by the Young-Laplace formula

$$
[p]=2 \sigma H
$$

where $\sigma$ is a constant for a given pair of fluids, and $H$ is the local mean curvature of the interface. The shape of the interface will adjust itself to meet this condition, and the outcome is a surface having minimal area consistent with the constraints; this is, in a sense, the smoothest such surface. We attempt to endow captured shocks with a property similar to this, which will reduce unnecessary area. This will involve first detecting the shocks, and then applying an artificial pressure difference across them.

\section{II.A. Recognizing shocks}

As a means of recognizing captured shocks, we examine the isobaric surfaces. In a mathematically ideal shockwave, the pressure gradient normal to the shock is infinite, and the tangential component is finite. The direction of the gradient is therefore normal to the shock, and this will be approximately true for captured shocks. There are no pressure gradients associated with other flow discontinuities such as shear surfaces or entropy discontinuities. Across these features the pressure is actually continuous. The proposed mechanism therefore uses closely spaced isobaric surfaces to indicate the presence of a shock, and the normals to the isobars to indicate its orientation. The proposal is to generate an artificial force in the direction of the pressure gradient, and having a magnitude proportional to the curvature of the isobaric surfaces. This strategy imitates the behavior of surface tension, which we now briefly recall.

\section{II.B. Surface Tension}

Consider a point $\mathrm{P}$ located in a curved surface. Erect the normal at P. Every plane $\Pi$ that contains $\mathrm{P}$ will intersect the surface in a curve, whose curvature is $\tau_{\Pi}$. The average value of $\tau_{\Pi}$ (which is also the average of the two principle curvatures, $\left(\tau_{\Pi}\right)_{\max },\left(\tau_{\Pi}\right)_{\min }$ which lie in perpendicular planes) is called the mean curvature $\tau$ of the surface

$$
H=\frac{1}{2}\left(\frac{1}{P O}+\frac{1}{P Q}\right)
$$

and this is the quantity that appears in the Young-Laplace formula. 


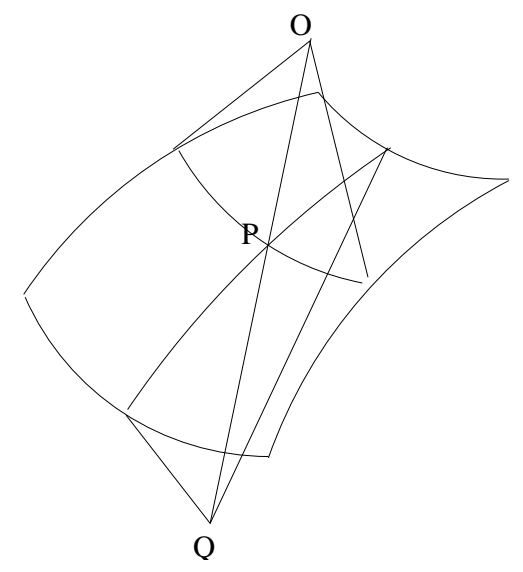

Figure 2. Illustrating the two principle curvatures of a saddle-shaped surface.

There is a very nice expression that gives the mean curvature of any family of surfaces that can be defined by giving their unit normal $\vec{n}$ as a function of position. This is

$$
H=\operatorname{div} \vec{n}
$$

Balabel et $a l^{1}$ have used this formula to calculate surface tension effects on droplets where $\vec{n}$ is the normal to a level set function. In our context the mean curvature of isobaric surfaces is

$$
H=\operatorname{div} \frac{\operatorname{grad} p}{|\operatorname{grad} p|}
$$

The proposal is to apply an artifical force to the fluid equal to

$$
\mathbf{F}=h \operatorname{gradp} H
$$

where $h$ is a small quantity with the dimensions of length. An obvious candidate is some measure of the local mesh size. To maintain the correct capturing of shocks, this force must be conservative, that is, it must be the divergence of some artificial flux tensor in the momentum equations. The following mathematical identity (whose proof is omitted) shows that the momentum flux tensor can be modified to give the desired correction.

$$
\operatorname{div}[|\operatorname{grad} p| \mathbf{I}-\vec{n} \otimes \operatorname{grad} p]=\operatorname{div} \vec{n} \operatorname{grad} p
$$

This produces a first-order scheme that is consistent in the sense of Lax and Wendroff. It also concentrates the force in the shock regions where grad $p$ is large. Choosing $k$ to be some nonlinear function of the gradient would give a higher-order scheme.

Modified in this way, the Euler equations still have the conservative form

$$
\frac{\partial \mathbf{u}}{\partial t}+\nabla \cdot \mathcal{F}=0
$$

but where now

$$
\mathcal{F}=\left(\begin{array}{c}
\rho \mathbf{v} \\
\rho \mathbf{v} \otimes \mathbf{v}+(p-k h|\operatorname{grad} p|) \mathbf{I}+k h \mathbf{n} \otimes \operatorname{grad} p \\
\mathbf{v}(E+p)
\end{array}\right)
$$

Evaluating the flux on an interface requires the values of the derivatives, and therefore the same stencil as a Navier-Stokes code. There is no tendency for this flux to broaden the shock.

It would be possible to use the modified pressure terms in the energy equation also. Experiments would be needed to see if this is worth doing. 


\section{II.C. A Model Situation}

Some insight into the proposed mechanism can be gained by appying it to a simple model pressure field. Consider a two-dimensional shock wave aligned with the y-axis, but subject to a sinusoidal perturbation. Near to the shock there is a pressure field defined by

$$
p=a x+b \cos y
$$

which represents a linearly increasing pressure with a sinusoidal perturbation in the transverse direction. For this case we would have

$$
\operatorname{grad} p=(a,-b \sin y)
$$

and

$$
\mathbf{n}=\frac{1}{\sqrt{a^{2}+b^{2} \sin ^{2} y}}(a,-b \sin y)
$$

The artificial flux tensor is this case is given by

$$
\mathbf{T}=\frac{k h}{\sqrt{a^{2}+b^{2} \sin ^{2} y}}\left(\begin{array}{cc}
b^{2} \sin ^{2} y & a b \sin y \\
a b \sin y & a^{2}
\end{array}\right)
$$

This leads to an artificial force given by

$$
\left(F_{x}, F_{y}\right)=\left(\frac{k h b \cos y}{\left(1+(b / a)^{2} \sin ^{2} y\right)^{3 / 2}}, \frac{-k h b^{2} \sin y \cos y}{a\left(1+(b / a)^{2} \sin ^{2} y\right)^{3 / 2}}\right)
$$

There are several instructive points to be noted about this example.

1. If the perturbations to the shock are small, so that $b / a<<1$ then $F_{y} / F_{x}<<1$ also.

2. Note that $\partial_{x} \mathbf{T}=0$ so that the force is due entirely to gradients in the $y$-direction. That is to say, on a Cartesisn grid, these forces would be produced by stresses on horizontal interfaces. There are tangential forces leading to $F_{x}$ and normal forces leading to $F_{y}$.

3. There will be some production of vorticity, which is frequently a concomitant of shock instability. This vorticity is in a helpful sense of rotation.

4. There is no force that tends to broaden the shock.

\section{Results and Speculations}

Preliminary results are shown in Figures 3 through 6 .

In Figures 3 and 4 the problem is a stationary shock in a parallel flow at $M=20$. The flux employed is the Roe flux with no entropy fix. This flux almost invariably fails all versions of this test. Here we incorporate AST with various values of the coefficient $\mathrm{k}$. Only one accurate solution is obtained, with $k=0.01$ after 500 time steps, but even this solution breaks up at later times.

The base flux in Figure 4 is a flux devised to be entropy consistent on all occasions. It has the form

$$
\mathbf{F}^{*}=\mathbf{F}_{C}-\frac{1}{2} \mathbf{R}\left(|\boldsymbol{\Lambda}|+\alpha|[\mathbf{\Lambda}]| \mathbf{R}^{T}[\mathbf{v}]\right.
$$

where $\mathbf{F}_{C}$ is a flux depending symmetrically on the left and right states, and constructed to conserve entropy. The vector of right eigenvectors is $\mathbf{R}$ and the diagonal matrix of eigenvectors is $\boldsymbol{\Lambda}$. With $\alpha=0$ this flux gives a scheme that always produces positive entropy at each interface, but not quite enough. The term containing $\alpha$ generates additional entropy wherever the wavespeed changes rapidly, that is to say at shocks and underresolved rarefactions. A value of 0.8 is larger than the theory would indicate. ${ }^{5}$ Although it succeeded in one dimensional tests, ${ }^{6}$ it failed totally in the "one-and-a half-dimensional" tests where one-dimensional problems were solved on two-dimensional grids. Here the combination of proper entropy production and AST is successful if $\mathrm{k}$ is chosen as either 0.5 or 1.0.

The flow in Figures 5 and 6 is (once again) that over a circular cylinder at a Mach number of 6.0 with $\gamma=1.4$. The grid is one that proved particularly troublesome in. ${ }^{6}$ It was formed by taking one 
circumferential grid line to coincide with the body and another to follow an accurately fitted shock. THe remaining circumferential lines were interpolated or extrapolated from these two. Paradoxically, aligning the grid with the shock in this way seems specially likely to induce instabilities, although many other details play a role. In Kitamura et $a l^{6}$ neither the original Roe flux nor the entropy consistent modification succeeded with this flow on this grid.

In Figure 5 a fairly good result is achieved with both fluxes. The exception is the oscillation near the top of the left figure. Quite possibly this is a bug in the code; certainly the AST terms can be satisfied with a symmetric solution.

It can definitely be concluded that Artificial Surface Tension succeeds in its objective of stabilising shock curvature. It is not surprising that this can only be achieved with a limited range of k. Certainly taking $k$ to be too small must be ineffective. On the other hand taking $k$ to be too big corresponds to an aggressive overcorrection that is also likely to fail. It is, however, a little disconcerting that the best values of $k$ are rather different for the two experiments. For the shock in parallel flow the best value seems to be about 0.5, whereas for the cylinder it is about 0.1 .

However, the two flows are at different Mach numbers, and it is possible that this is more important than the geometry. More research is required to find reliable values of $k$. Meanwhile, Artificial Surface Tension is offered as another weapon to those who find themselves battling with strange results from their Euler codes.

\section{Acknowledgements}

The work of the first author was supported in part by the Space Vehicles Technology Institute under grant NCC3-589 funded jointly by NASA and DOD with the Constellation University Intitute Project, with Claudia Myer as the projects manager.

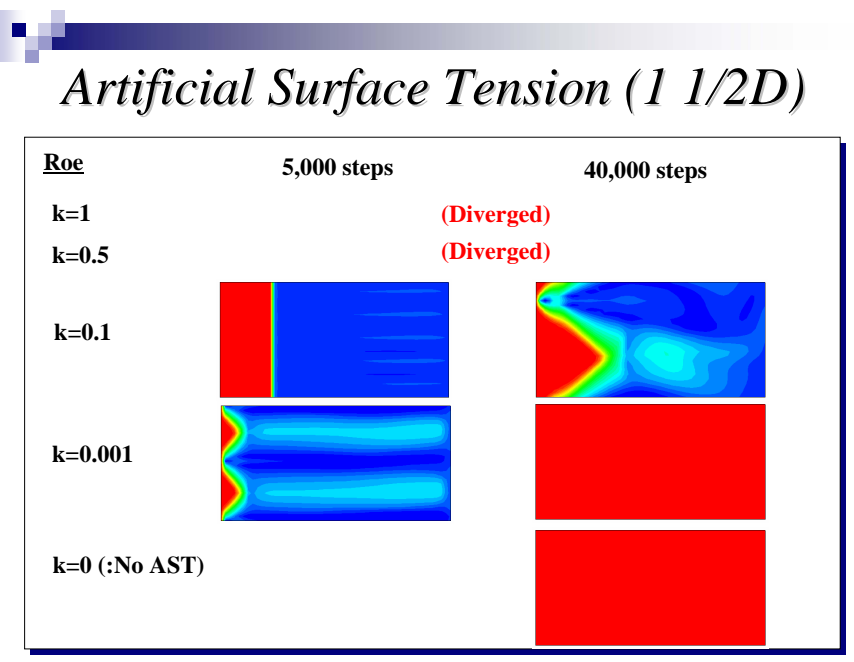

Figure 3. Results for a stationary shockwave in a parallel flow. The base scheme is the original Roe scheme. A solid red image indicates that the shock has left the domain.

\section{References}

\footnotetext{
${ }^{1}$ Balabel,A., Binninger, B., Herrmann, N., Peters, N., Calculation of droplet deformation by surface tension effects using level set method. Combustion Science and Technology, 174, 2002.

${ }^{2}$ Barth, T. J., Some notes on shock-resolving flux functions, part 1: Stationary characteristics. NASA TM 101087, Ames Research Center, 1989.

${ }^{3}$ Bultelle, M., Grassin, M., Serre, D., Unstable Godunov discrete profiles for steady shock waves. SIAM J. Num. Anal. 35 (1998).

${ }^{4}$ Dumbser, M., Moschetta, J. M., Gressier, J., A matrix cstability analysis of the carbuncle phenomenon, J. Comp. Phys., 197, 2004.

${ }^{5}$ Ismail, F, Roe, P. L., Affordable, entropy-consistent, Euler flux functions, II Entropy production at shocks, in preparation.
} 


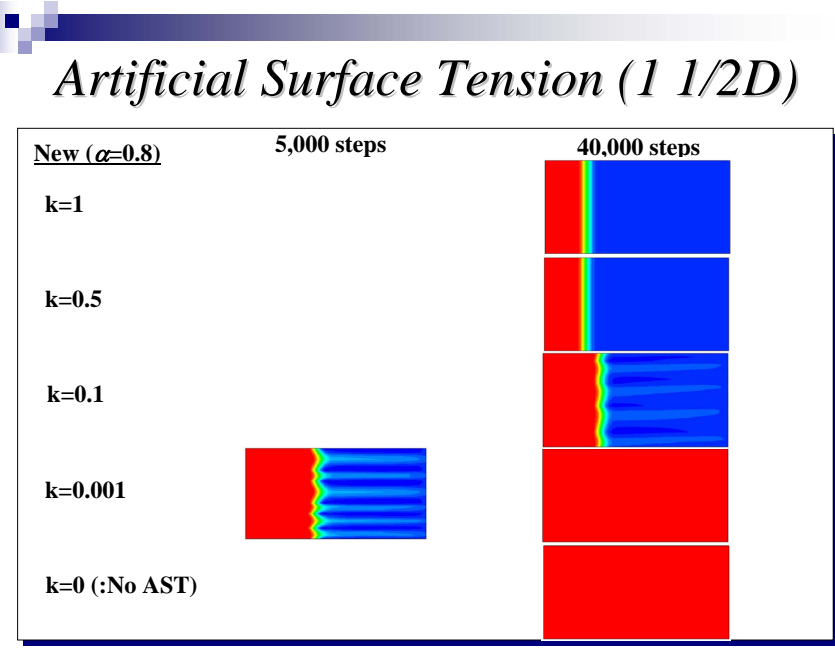

Figure 4. Results for a stationary shockwave in a parallel flow. The base scheme is the entropy-consistent Roe scheme. A solid red image indicates that the shock has left the domain.
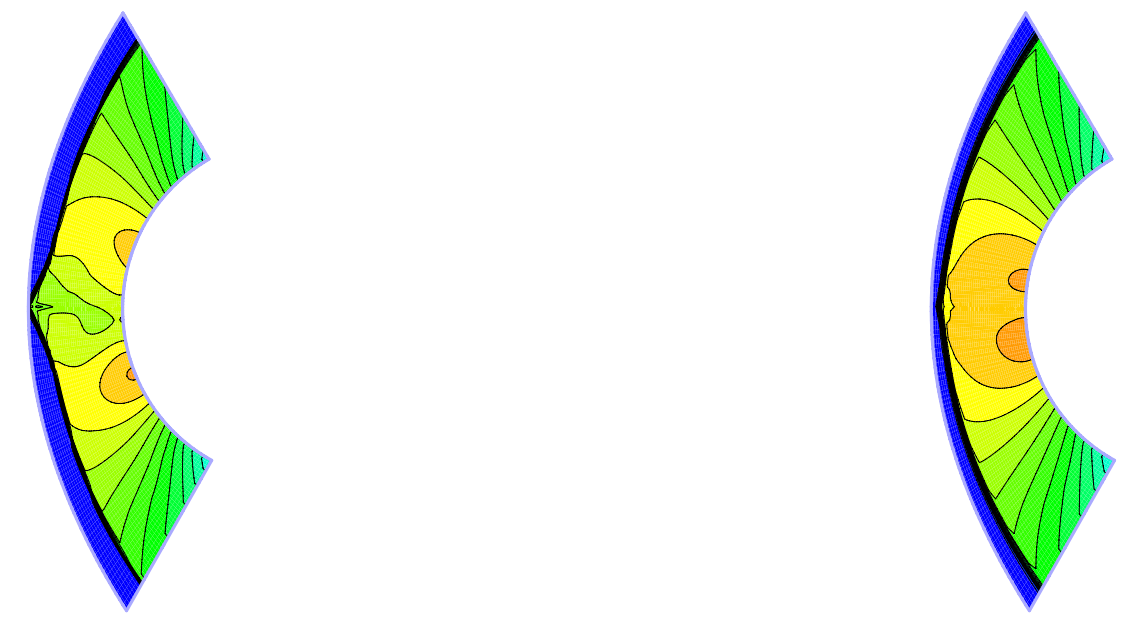

Figure 5. Flow at $M=6$ past a circular cylinder; left, original Roe flux; right, entropy-consistent flux. No Ast

${ }^{6}$ Kitamura, K., Roe, P. L. Ismail, F., An Evaluation of Euler Fluxes for Hypersonic Flow Computations, AIAA Paper 2007-4465I, 18th AIAA Computational Fluid Dynamics Conference, Miami, FL, Jun. 2007.

${ }^{7}$ Maull,D. J., Hypersonic flow over axially symmetric spiked bodies, J. Fluid Mech., 8, 1960.

${ }^{8}$ Menezes,V, Saravanan, S., Reddy K. P. J., Shock tunnel study of spiked aerodynamic bodies flying at hypersonic Mach numbers, Shock Waves, 12, 2002.

${ }^{9}$ Pandolfi, M., d'Ambrosio, D., Numerical Instablilities in Upwind Methods: Analysis and Cures for the Carbuncle Phenomenon J Comp. Phys. 166(2), 2001)

${ }^{10}$ Peery, K. M., Imlay, S. T., Blunt body flow simulations, it AIAA paper 88-2924, 1988.

${ }^{11}$ Quirk, J. J., A contribution to the great Riemann solver debate, Int. J. Num. Meth. Fluids, 32, 1994.

${ }^{12}$ Quirk, J. J., An Adaptive Grid Algorithm for Computational Shock Hydrodynamics, Ph.D thesis, College of Aeronautics, Cranfield Institute of Tech, 1991

${ }^{13}$ Whitham, G. B., Linear and Nonlinear Waves, Wiley, 1974. 

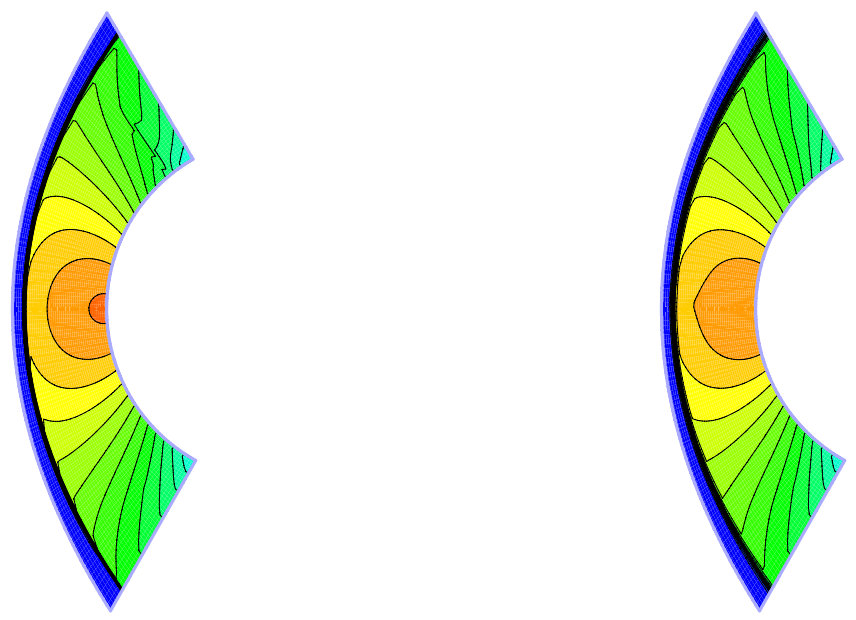

Figure 6. Flow at $M=6.0$ past a circular cylinder. left, original Roe flux; right, entropy-consistent flux. AST added with $k=0.1$ 